\title{
Supporting information: \\ Multifold enhancement of third-harmonic generation in dielectric nanoparticles driven by magnetic Fano resonances
}
A. S. Shorokhov ${ }^{1}$, E. V. Melik-Gaykazyan ${ }^{1}$, D. A. Smirnova ${ }^{2}$, B. Hopkins ${ }^{2}$, K. E. Chong ${ }^{2}$, D.-Y. Choi ${ }^{3}$, M. R. Shcherbakov ${ }^{1}$, A. E. Miroshnichenko ${ }^{2}$, D. N. Neshev ${ }^{2}$, A. A. Fedyanin ${ }^{1}$, and Y. S. Kivshar ${ }^{2}$
${ }^{1}$ Faculty of Physics, Lomonosov Moscow State University, Moscow 199991, Russia
${ }^{2}$ Nonlinear Physics Centre, Australian National University, Canberra ACT 2601, Australia
${ }^{3}$ Laser Physics Centre, Australian National University, Canberra ACT 2601, Australia

(Dated: July 14, 2016)

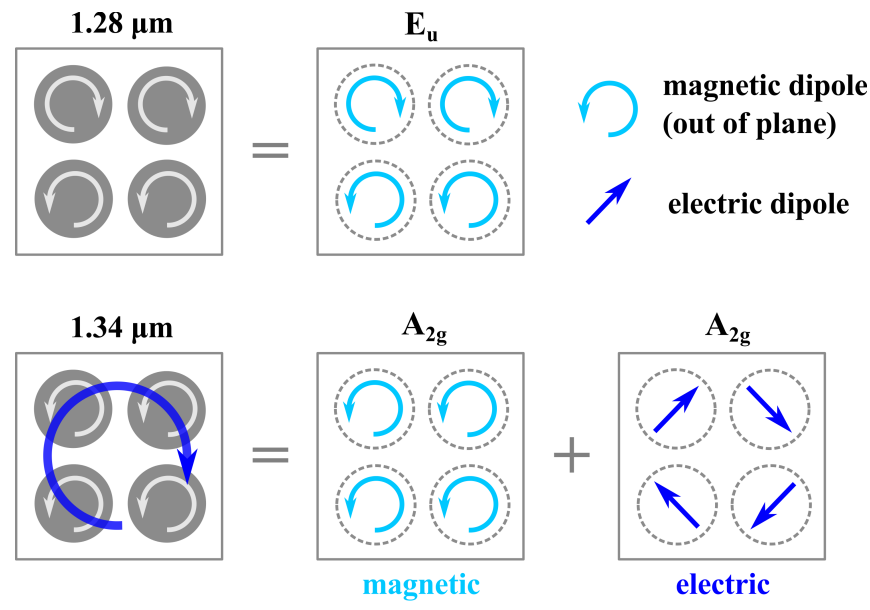

FIG. S1. $\mathrm{E}_{u}$ and $\mathrm{A}_{2 g}$ modes in a silicon nanodisks quadrumer illustrated schematically in the coupled electric and magnetic dipole approximation.

\section{COUPLED ELECTRIC AND MAGNETIC DIPOLE APPROXIMATION}

As discussed in the main text, we attribute two dips in the linear transmission spectrum to the dominant resonant excitation of different collective modes in the quadrumers. The first resonance is associated with the $\mathrm{E}_{u}$ magnetic mode, which is formed by four out-of-plane magnetic dipoles being out-of-phase for top and bottom disks of the quadrumer $[y$ axis is the vertical axis in the figures], whereas the second resonance is formed by the interaction between two strongly coupled $\mathrm{A}_{2 g}$ (electric and magnetic) modes (see schematics in Figure S1 and Ref. [1] for more details). The magnetic $\mathrm{A}_{2 g}$ mode is composed of four out-of-plane magnetic dipoles being in-phase. The electric $\mathrm{A}_{2 g}$ mode corresponds to the azimuthal arrangement of in-plane electric dipoles, which constitute a circular current over four disks of the quadrumer.

We can elucidate the near-field interaction between the $\mathrm{A}_{2 g}$ modes, using a simple coupled dipole model. Accordingly, the quadrumer can be considered as a system of electric and magnetic dipoles, as illustrated in Figure S1. The electric $\mathbf{E}$ and magnetic $\mathbf{H}$ fields created

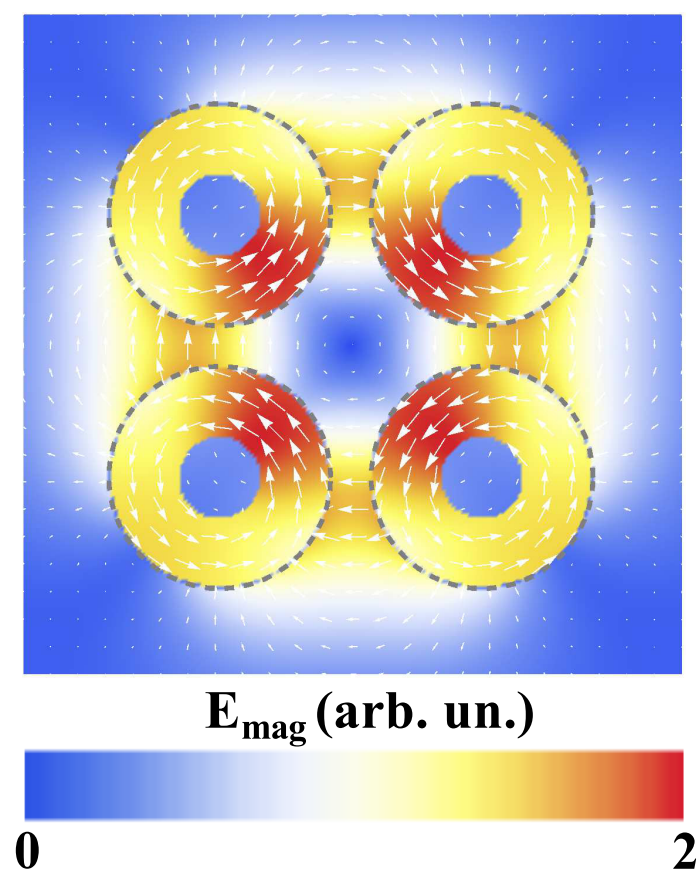

FIG. S2. The distribution of the electric field magnitude in the quadrumer, calculated using the coupled electric and magnetic dipole model.

by arbitrarily oriented electric $\mathbf{p}$ and magnetic $\mathbf{m}$ point dipoles located at positions $\mathbf{r}^{p}$ and $\mathbf{r}^{m}$, respectively, are found from the following equations [2]:

$$
\begin{array}{r}
\mathbf{E}^{p}(\mathbf{r})=\frac{k_{0}^{2}}{\varepsilon_{0}} \hat{G}\left(\mathbf{r}, \mathbf{r}^{p}\right) \mathbf{p} \\
\mathbf{H}^{p}(\mathbf{r})=\frac{k_{0} c}{i} \nabla \times \hat{G}\left(\mathbf{r}, \mathbf{r}^{p}\right) \mathbf{p} \\
\mathbf{H}^{m}(\mathbf{r})=k_{d}^{2} \hat{G}\left(\mathbf{r}, \mathbf{r}^{m}\right) \mathbf{m} \\
\mathbf{E}^{m}(\mathbf{r})=\frac{i k_{0}}{c \varepsilon_{0}} \nabla \times \hat{G}\left(\mathbf{r}, \mathbf{r}^{m}\right) \mathbf{m}
\end{array}
$$

Here $k_{0}$ is the wave number in vacuum, $\varepsilon_{0}$ is the vacuum permittivity, $c$ is the vacuum speed of light, $k_{d}$ is the wave number in the dielectric medium. The Green's tensor $\hat{G}$ 
in the medium is given by

$$
\begin{gathered}
\hat{G}\left(\mathbf{r}_{l}, \mathbf{r}_{j}\right)=\left[\left(\frac{1}{R_{l j}}+\frac{i}{k_{d} R_{l j}^{2}}-\frac{1}{k_{d}^{2} R_{l j}^{3}}\right) \hat{U}+\right. \\
\left.+\left(-\frac{1}{R_{l j}}-\frac{3 i}{k_{d} R_{l j}^{2}}+\frac{3}{k_{d}^{2} R_{l j}^{3}}\right) \mathbf{e}_{\mathbf{R}} \mathbf{e}_{\mathbf{R}}\right] \frac{e^{i k_{d} R_{l j}}}{4 \pi},
\end{gathered}
$$

where $R_{l j}=\left|\mathbf{R}_{l j}\right|=\left|\mathbf{r}_{l}-\mathbf{r}_{j}\right|, \mathbf{e}_{\mathbf{R}} \mathbf{e}_{\mathbf{R}}$ is the dyadic constructed from the unit vector $\mathbf{e}_{\mathbf{R}}=\mathbf{R}_{l j} / R_{l j}, \hat{U}$ is the unit $3 \times 3$ tensor. As was already mentioned, our system consists of four magnetic dipoles co-oriented out of the sample plane and four electric dipoles excited in the plane (see Figure S1). Therefore, we write the electric field outside of the disks, forming the quadrumer, as follows:

$$
\begin{array}{r}
\mathbf{E}_{\text {out }}(\mathbf{r})=\sum_{j=1}^{4} \mathbf{E}_{j}^{p}+\sum_{j=1}^{4} \mathbf{E}_{j}^{m}= \\
=\sum_{j=1}^{4} \frac{k_{0}^{2}}{\varepsilon_{0}} \hat{G}\left(\mathbf{r}, \mathbf{r}_{j}^{p}\right) \mathbf{p}_{j}+\sum_{j=1}^{4} \frac{i k_{0}}{c \varepsilon_{0}} \nabla \times \hat{G}\left(\mathbf{r}, \mathbf{r}_{j}^{m}\right) \mathbf{m}_{j},
\end{array}
$$

while inside the $n$th disk the electric field is expressed as

$$
\begin{aligned}
& \mathbf{E}_{\mathrm{in}}^{(n)}(\mathbf{r})=\mathbf{E}_{0}^{(n)}+\sum_{j \neq n}^{4} \mathbf{E}_{j}^{p}+\sum_{j \neq n}^{4} \mathbf{E}_{j}^{m}=\mathbf{E}_{0}^{(n)}(\mathbf{r})+ \\
& +\sum_{j \neq n}^{4} \frac{k_{0}^{2}}{\varepsilon_{0}} \hat{G}\left(\mathbf{r}, \mathbf{r}_{j}^{p}\right) \mathbf{p}_{j}+\sum_{j \neq n}^{4} \frac{i k_{0}}{c \varepsilon_{0}} \nabla \times \hat{G}\left(\mathbf{r}, \mathbf{r}_{j}^{m}\right) \mathbf{m}_{j} .
\end{aligned}
$$

Here $\mathbf{E}_{0}^{(n)}$ is the local electric field distribution inside the $n$th disk excited by the incident radiation without influence from the other disks.

Applying this model, we calculate the electric field distribution resulting from the interplay between two $\mathrm{A}_{2 g}$ modes, as shown in Figure S2. The accepted approximation is found to be quite accurate in emulating the results of full-wave simulations.

\section{NUMERICAL SIMULATIONS FOR AN ARAY OF SINGLE NANODISKS}

To evidence the benefit of using the quadrumers instead of the uniformly spaced disks for nonlinear dielectric metasurfaces, we perform an additional set of numerical calculations with FEM solver of COMSOL Multiphysics. We simulate a periodic array of a-Si:H nanodisks excited by the s-polarized plane wave incident at a $45^{\circ}$ angle. The diameter and the height of the disks are the same as those in Figure 3 of the main text. Here, however, the period is chosen twice smaller than the lattice constant of the array of quadrumers so that the unit cell occupies the quarter area, in order to maintain the same

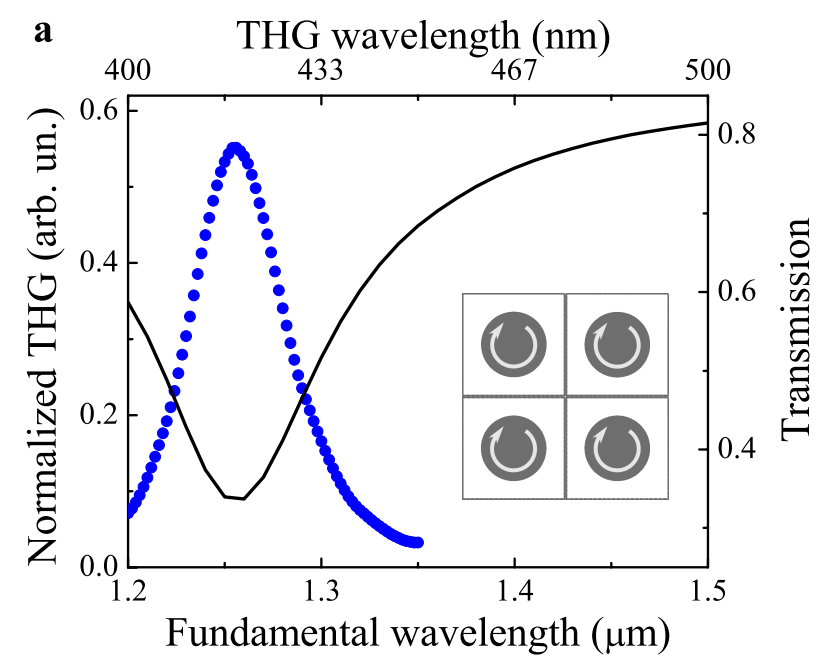

b
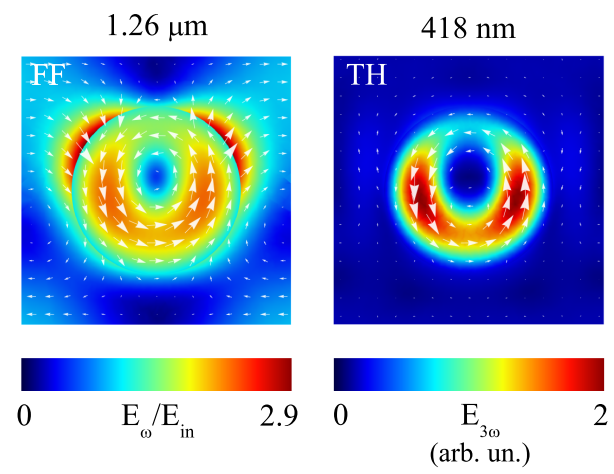

FIG. S3. (a) TH emission power (blue dots) overlaid with the linear transmission spectrum (black curve) calculated numerically for a periodic array of a-Si:H nanodisks excited by the s-polarized plane wave incident at a $45^{\circ}$ angle. (b) Normalized FF (left) and TH (right) electric field distribution superimposed with field vectors at the fundamental wavelength $1.255 \mu \mathrm{m}$ corresponding to the peak $\mathrm{TH}$ power. The fields are plotted in the horizontal $x y$ cut-plane through the disk center over one unit cell. The incident field intensity, disk sizes and normalization of the plotted quantities are the same as those in Figure 3 of the main text. The period is chosen twice smaller than the lattice constant of the array of quadrumers, in order to maintain the same filling factor of the nonlinear material.

filling factor of the nonlinear material. Results of the calculations are presented in Figure S3. The linear transmission spectrum (black line) indicates a pronounced dip, which can be attributed to the excitation of the magnetic dipolar resonance in the nanodisk (see the corresponding near-field distribution at the fundamental wavelength in Figure S3). The enhancement of the local electric field within the disks in the vicinity of the magnetic resonance leads to the increase in the TH radiated power (shown with blue dots). Nonetheless, this enhancement is notably smaller than in the case of quadrumers. 


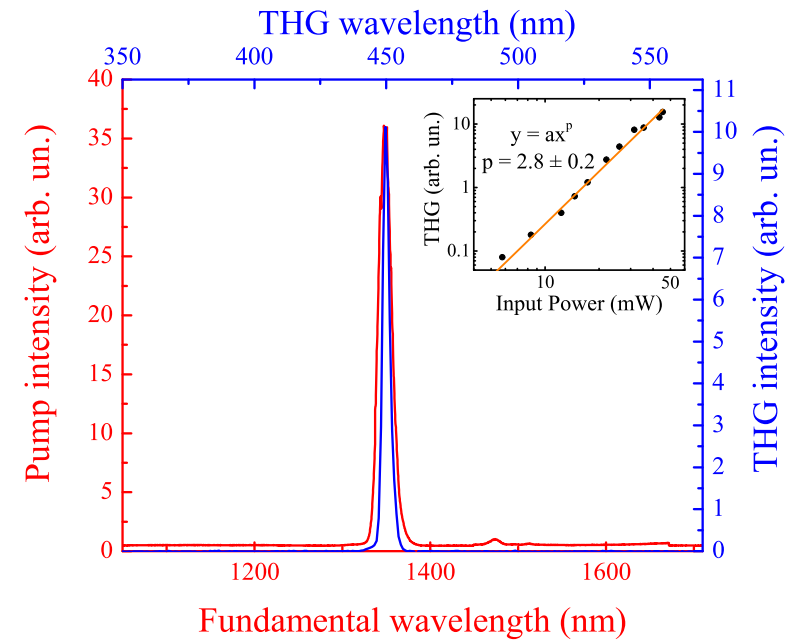

FIG. S4. Experimental spectra of the pump laser (red) and TH (blue) signal. Inset shows the dependence of the TH intensity on the incident laser power (black dots); orange line depicts the fit of the experimental data with a function $y=a x^{p}$, resulting in the power $p=2.8 \pm 0.2$.

\section{ADDITIONAL EXPERIMENTAL DATA}

In order to prove the THG nature of the observed signal, we measured spectra of both the pump laser and the detected radiation. Experimental results are shown in Figure S4. The pump spectrum was measured with a Solar-LS M266 grating spectrometer ended by a convection-cooled Hamamatsu InGaAs CCD linear array. The spectrum of the signal was obtained using a SolarLS S100 concave grating spectrometer. The TH origin of the signal was also confirmed by checking the cubic dependence of its intensity on the pump power (see inset in Figure S4). Fitting the experimental data with the power-law function $y=a x^{p}$ gives the factor $p$ of about $2.8 \pm 0.2$. Estimated THG conversion efficiency of the quadrumers sample was up to $10^{-6}$, which is 10 times larger than in the previous study of an array of opticallydecoupled silicon nanodisks [3].

* Corresponding author: ysk@internode.on.net

[1] Hopkins, B.; Filonov, D. S.; Miroshnichenko, A. E.; Monticone, F.; Alú, A.; Kivshar, Y. S. ACS Photonics 2015, 2, 724-729.

[2] Evlyukhin, A. B.; Reinhardt, C.; Seidel, A.; Luk'yanchuk, B. S.; Chichkov, B. N. Phys. Rev. B 2010, 82, 045404.

[3] Shcherbakov, M. R.; Neshev, D. N.; Hopkins, B.; Shorokhov, A. S.; Staude, I.; Melik-Gaykazyan, E. V.; Decker, M.; Ezhov, A. A.; Miroshnichenko, A. E.; Brener, I.; Fedyanin, A. A.; Kivshar, Y. S. Nano Lett. 2014, 14, 6488-6492. 